Medizinprodukte

\title{
Studienevidenz nach der Zulassung sehr unterschiedlich
}

\author{
Vor der Zulassung müssen die Hersteller von Hochrisiko- \\ Medizinprodukten deren Sicherheit und Wirksamkeit \\ nachweisen. In den USA werden klinische Daten zunehmend \\ jedoch auch durch kontinuierliche Anwendungsstudien nach \\ der Markteinführung gewonnen. Die Evidenz dieser Studien \\ wurde nun unter die Lupe genommen. \\ JAMA 2015; 314: 604-612
}

Laut Definition unterstützen oder erhalten Hochrisiko-Medizinprodukte (MP) das Leben des Patienten und beugen Krankheiten oder potenziellen Risiken vor. In den USA werden solche MPs üblicherweise über das sog. Premarket-Approval-Verfahren (PMA) der US-amerikanischen Arzneimittelbehörde FDA zugelassen. Hierfür muss der Hersteller vor der Zulassung die Sicherheit und Wirksamkeit des Produkts nachweisen. Zunehmend werden Hochrisiko-MPs aber auch während ihrer ganzen Lebensdauer getestet. In einer amerikanischen Studie wurden nun die Studienmerkmale dafür überprüft.
Hierzu untersuchten die Autoren die Daten von Hochrisiko-MPs, die zwischen Januar 2010 und Dezember 2011 über das PMA-System zugelassen worden waren. Dabei bedienten sie sich der öffentlichen PMA-Datenbank. Die Produkte wurden wie folgt charakterisiert:

- Zulassungsjahr,

- medizinisches Einsatzgebiet,

- Art des Gutachtens (normal bzw. beschleunigt),

- Implantations-Kennzeichnung (ja oder nein).
Ferner wurde erfasst, ob es sich um

- Studien vor der Markteinführung

(Pilot- oder pivotale Studien) oder

- Anwendungsstudien (durch FDA bzw. den Hersteller / Prüfarzt initiiert)

handelte.

Im Untersuchungszeitraum wurden 28 Hochrisiko-MPs per PMA zugelassen. Die 286 erfassten Studien waren meist Anwendungsstudien nach der Zulassung (71,3\%). Der Anteil der Hersteller-initiierten Studien lag bei 59,8\%. Die mediane Probandenzahl lag zwischen 65 (Pilotstudien vor der Zulassung) und 250 (Hersteller-initiierte post-Zulassungsstudien). Etwa die Hälfte aller Studien nutzte keine Vergleichsparameter. Die mediane Nachbeobachtungszeit betrug

- 3 Monate für Zulassungsstudien,

- 9 Monate für abgeschlossene und

- 12 Monate für noch laufende post-Zulassungsstudien.

\section{Fazit}

Den Autoren zufolge variiert die Studienevidenz von Hochrisiko-MPs stark. Die Studie bestätigt frühere Ergebnisse, wonach Zulassungsstudien für MPs häufig nur eingeschränkte Daten liefern.

Dr. Marion Rukavina, Berlin

Kommentar aus der Praxis

Das amerikanische Premarket-ApprovalVerfahren (PMA) der Food-and-Drug-Administration (FDA) gilt allgemein als das weltweit strengste Regulierungssystem für Medizinprodukte (MP). Dieses zentrale Zulassungsverfahren kommt seit Ende der 1970er Jahre in den USA für Hochrisiko-MPs zum Einsatz.

Ähnlich den Anforderungen für den Marktzugang von neuen Arzneimitteln, verlangt die FDA von MP-Herstellern präklinische und vor allem klinische Daten. Diese Daten sollen die Sicherheit und Wirksamkeit eines MP in der beantragten Indikation belegen. Spätestens seit dem FDA-Modernization-Act von 1997 verwässern jedoch die ehemals hohen Anforderungen an die zu erbringenden Nachweise wie etwa an das zugrunde zulegende Studiendesign.
Die Entwicklungen in der jüngeren Vergangenheit gehen sogar soweit, dass der Erhebung von klinischen Daten nach der Zulassung ein größerer Stellenwert beigemessen wird - mit dem Ziel, neue MPs für Patienten schnell zugänglich zu machen und gleichzeitig Produktinnovationen den Marktzugang zu erleichtern.

Die Untersuchung von Rathi et al. wäre im europäischen - und damit auch deutschen - MP-Marktzugangssystem aufgrund der bestehenden Intransparenz so nicht möglich. Anstelle eines VorherNachher-Vergleichs stellt sie allerdings lediglich eine Momentaufnahme der Studienquantität und -qualität im Rahmen ausgewählter PMA-Verfahren dar.

Zudem wirft die Studie die ein oder andere methodische Frage auf: Bspw. könnte die Generierung des Datensatzes oder die Einteilung der Endpunkte dazu geführt haben, dass die tatsächliche Studienqualität überschätzt wurde.

Davon abgesehen verdeutlicht diese Untersuchung aufs Neue, dass sich Ärzte über die qualitative und quantitative Variabilität der klinischen Evidenz zu neu auf dem Markt verfügbaren MP im Klaren sein müssen. Zudem zeigt sie, dass auch eine Zulassung in den USA keine Garantie für bestmögliche Studiendaten ist. Diese Fakten müssen berücksichtigt werden, wenn Patienten über mögliche Vor- und Nachteile eines MP aufgeklärt werden.

Dipl.-Oecotroph. MPH Yvonne Zens Institut für Qualität und Wirthschaftlichkeit im Gesundheitswesen (IQWiG), Köln 